\title{
Dynamic X-ray Imaging at the Munich Compact Light Source
}

$\underline{\text { Regine Gradl }}^{1,2, *}$, Martin Dierolf ${ }^{1}$, Lorenz Hehn ${ }^{1,3}$, Benedikt Günther ${ }^{1,4}$, David Kutschke ${ }^{5,6}$, Lin Yang ${ }^{5,6}$, Winfried Möller ${ }^{5,6}$, Tobias Stoeger ${ }^{5,6}$, Melanie Kimm ${ }^{3}$, Helena Haas ${ }^{3}$, Nathalie Roiser ${ }^{3}$, Daniela Pfeiffer $^{3}$, Martin Donnelley ${ }^{7,8}$, Christoph Jud ${ }^{1}$, Bernhard Gleich ${ }^{1}$, David Parsons ${ }^{7,8}$, Klaus Achterhold ${ }^{1}$, Otmar Schmid ${ }^{5,6}$, Franz Pfeiffer ${ }^{1,2,3}$ and Kaye S. Morgan ${ }^{1,2,9}$

${ }^{1 .}$ Chair of Biomedical Physics and Munich School of BioEngineering, Technical University of Munich, Garching, Germany

2. Institute for Advanced Studies, Technical University of Munich, Garching, Germany

3. Department of Diagnostic and Interventional Radiology, Klinikum rechts der Isar, Technical University of Munich, München, Germany

4. Max-Plank-Institute for Quantum Optics, Garching, Germany

5. Comprehensive Pneumology Center, Member of the German Center for Lung Research (DZL), München, Germany

6. Institute of Lung Biology and Disease, Helmholtz Zentrum München, Neuherberg, Germany

7. Robinson Research Institute, University of Adelaide, Adelaide, Australia

8. Respiratory and Sleep Medicine, Women's and Children's Hospital, Adelaide, Australia

9. School of Physics and Astronomy, Monash University, Clayton, Australia

* Corresponding author, regine.gradl@ tum.de

In vivo dynamic respiratory phase-contrast x-ray imaging (PCXI) in small animal models is a powerful and relatively new research tool, which can be used to increase physiological understanding, and accelerate new therapies towards the clinics. Phase-contrast techniques create strong contrast from airtissue interfaces, rendering visible airway structures that suffer from low contrast in conventional absorption imaging. To make use of phase contrast, and capture high-speed image sequences, high flux and high beam coherence are needed. Therefore, biomedical research studies, like the assessment of new treatments for cystic fibrosis airway disease [1], or the development of neonatal ventilation strategies [2], were previously limited to synchrotron facilities. Here we present the first dynamic in vivo x-ray phase-contrast imaging results obtained in a laboratory environment, using a compact light source.

The Munich Compact Light Source (MuCLS) is bridging the gap between conventional laboratory sources and large-scale synchrotron facilities. With a footprint of about $5 \mathrm{~m} \times 3 \mathrm{~m}$, the MuCLS generates brilliant, partially coherent and quasi-monochromatic x-rays, anywhere between $15 \mathrm{keV}$ and $35 \mathrm{keV}$. This is achieved by inverse Compton scattering of infrared laser photons with relativistic electrons [3,4]. The beam divergence of $4 \mathrm{mrad}$ is sufficiently low to enable long sample-to-detector distances for propagation-based phase contrast [5], and the high flux (up to $3 \times 10^{10}$ photons/s) facilitates dynamic imaging.

Two different opportunities for dynamic phase-contrast imaging have been realized at this source. One is grating-based imaging focusing on repeated motion (e.g. the breath cycle of a mouse) and the other is propagation-based imaging for irreversible changes (e.g. testing the effects and distributions of treatments). We show that the MuCLS can produce high-visibility propagation-based phase-contrast images at exposure times as low as $50 \mathrm{~ms}$, which is sufficient to track respiratory processes $[5,6]$.

Fig. 1 and Fig. 2 show two examples of dynamic propagation-based imaging, where we used the phase 
effects to enhance the contrast of the lungs and airways, capturing the delivery of treatment and the clearance of inhaled particles along the airway surface. Fig. 1 displays images from a time sequence capturing a $50 \mu \mathrm{l}$ liquid instillation into the lung. In this case, the liquid is distributed quite locally, however in the same study a range of different liquid distributions were observed (with the same liquid volume). These kinds of studies enable a previously-inaccessible high-resolution, time-resolved insight into the distribution of treatments, and help to answer questions why treatments are distributed in different ways. Fig. 2 shows inhaled debris sitting on the surface of airways in the lower respiratory system. This debris is normally transported along the airways towards the mouth by mucociliary transit (MCT), an essential mechanism for maintaining healthy lungs. However, in some diseases, like cystic fibrosis, MCT is inhibited. Dynamic x-ray imaging offers the possibility to non-invasively quantify individual particle MCT. This means that these measurements can be made following delivery of treatments to assess their efficacy, e.g. by analyzing the clearance speed of the debris. In both of these examples, previous in vivo research has relied upon a low-resolution fluorescence signal.

The detailed images presented here, captured with high time and spatial resolution, demonstrate what is possible in this research field. This presentation will describe these and further applications, and detail the current and future capabilities of this laboratory-based imaging set-up [7].

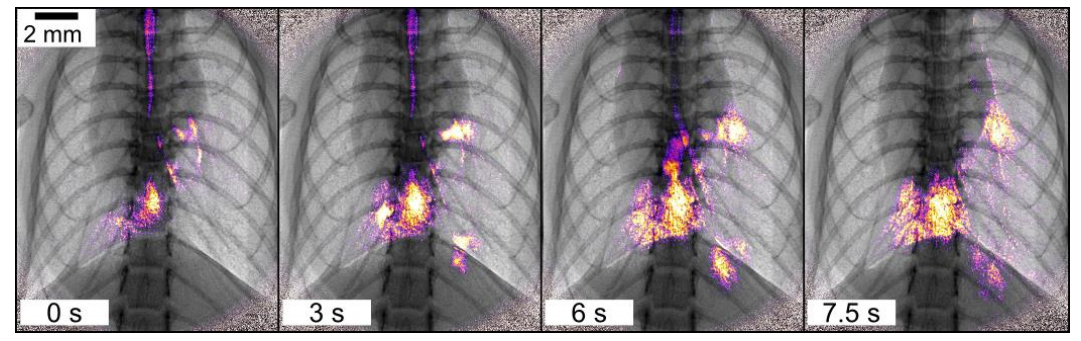

Figure. 1. Pseudo-colored images of a $50 \mu \mathrm{l}$ liquid instillation, used to mimic respiratory treatment delivery. In this case the liquid is locally distributed. The timepoints are in reference to the first image in the figure. (1.33 fps, exposure time $200 \mathrm{~ms})$
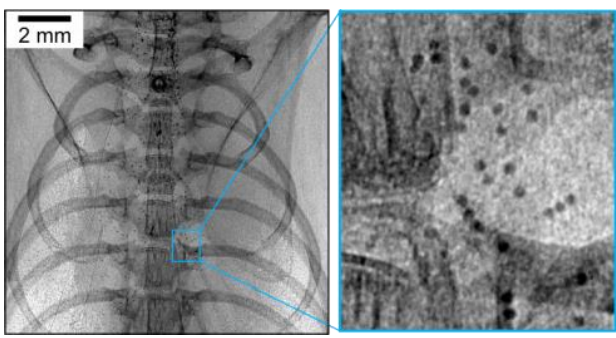

Figure. 2. Mucociliary clearance can be observed by tracking small debris in the airways, shown here using $75 \mu \mathrm{m}$ beads.

[1] K. S. Morgan et al, Am. J. Respir. Critical Care Medicine 190 (2016), 469.

[2] A. B. Te Pas et al, Pediatr. Res. 65 (2009), 537.

[3] The MuCLS machine was designed and constructed by Lyncean Technologies Inc. (Fremont, USA), http://lynceantech.com/.

[4] E. Eggl et al, J. of Synchrotron Radiation 23 (2016), 1313.

[5] R. Gradl et al, Scientific Reports 7 (2017), 42211.

[6] R. Gradl et al, under review (2018).

[7] We acknowledge financial support through the Centre for Advanced Laser Applications (CALA), and the DFG Gottfried Wilhelm Leibniz program. KM was supported by a Veski VPRF. KM, RG and FP completed this work with the support of the TUM Institute for Advanced Study, funded by the German Excellence Initiative and the European Union Seventh Framework Program under grant agreement no 291763 and co-funded by the European Union. LY received a fellowship from China Scholarship Council (CSC). MDo supported by NHMRC project APP1079712, and by the Cure 4 Cystic Fibrosis Foundation. 